Sociologie et sociétés

\title{
Intimité conjugale et intimité personnelle
}

À la recherche d'un équilibre entre deux exigences dans les

sociétés modernes avancées

\section{Intimacy in Married and Personal Life}

\section{In Search of a Balance Between Two Demands in Advanced Modern Societies}

\section{François De Singly}

Volume 35, numéro 2, automne 2003

De l'intimité

Intimacy: Several Issues

URI : https://id.erudit.org/iderudit/008524ar

DOI : https://doi.org/10.7202/008524ar

Aller au sommaire du numéro

Éditeur(s)

Les Presses de l'Université de Montréal

ISSN

0038-030X (imprimé)

1492-1375 (numérique)

Découvrir la revue

Citer cet article

De Singly, F. (2003). Intimité conjugale et intimité personnelle : à la recherche d'un équilibre entre deux exigences dans les sociétés modernes avancées. Sociologie et sociétés, 35(2), 79-96. https://doi.org/10.7202/008524ar

\section{Résumé de l'article}

Il s'agit dans cet article de soumettre à la critique, à la fois théorique et empirique, le modèle de la "relation pure ", proposé par Giddens comme forme idéale de la vie à deux dans les sociétés modernes avancées. Ce modèle sous-estime nettement le besoin de sécurité ontologique des individus, même les plus « individualisés ». La fragilité de ces derniers réclame certaines conditions, et notamment des preuves de reconnaissance de soi qui s'inscrivent dans la demande - qui reste très forte - de fidélité, ou encore de temps passé ensemble. Une enquête par questionnaire passé auprès de membres de couples de la région parisienne et d'un certain niveau scolaire permet de montrer la force des compromis entre la " pureté » des libertés individuelles au sein de la vie conjugale et les contraintes de la reconnaissance de chacun des deux partenaires. La vie commune n’a donc pas nécessairement comme avenir « la relation pure" 


\section{Intimité conjugale et intimité personnelle}

À la recherche d'un équilibre entre deux exigences dans les sociétés modernes avancées

\section{FRANÇOIS DE SINGLY}

Centre de recherches sur les liens sociaux CNRS - Université de Paris 5

45 , rue des Saints-Pères

75006 Paris, France

Courriel : francois@singly.org.

\section{LES TROIS PÉRIODES DE L'INTIMITÉ}

T 'histoire de la Vie privée en Occident permet d'appréhender les transforma— tions de l'intimité (Hall, 1987). Pendant la première étape — à partir du milieu du XVIII ${ }^{\mathrm{e}}$ siècle selon Philippe Ariès (1960) —, la famille se sépare du reste de la société, des voisins, de la parenté, du monde du travail. Le cercle de famille se forme en se resserrant autour du père, de la mère et des enfants. Les domestiques ne dorment plus dans le même logement que leurs employeurs (Eleb-Vidal et Debarre-Blanchard, 1989). À la fin du $\mathrm{XIX}^{\mathrm{e}}$ siècle, commence une deuxième période: celle de la spécialisation plus grande des pièces, notamment avec les chambres des enfants et la chambre conjugale. Selon Alain Corbin (1987), la sexualité de plus en plus conjugale s'inscrit dans une chambre commune, contrairement aux générations antérieures. Progressivement, les plus jeunes accèdent à une chambre individuelle. Ils ont ce droit à un espace privé à l'intérieur même du logement familial pour apprendre ainsi à quitter leurs parents, à devenir autonomes. L'intimité «familiale» coexiste alors avec deux autres types d'intimité: «personnelle» pour les enfants, «conjugale» pour les adultes.

À partir du milieu du $x^{\mathrm{e}}$ siècle, sous l'influence à la fois de la scolarisation des jeunes filles et femmes et du mouvement féministe, naît une troisième période pendant laquelle les membres du couple revendiquent une certaine intimité personnelle, 
c'est-à-dire le droit à avoir une vie indépendante de leur conjoint et autonome, au moins en partie. L'intimité familiale abriterait désormais une distinction supplémentaire, l'intimité «personnelle» pour les femmes comme pour les hommes (ces derniers ont eu accès, avant elles au $\mathrm{XIX}^{\mathrm{e}}$ siècle, à cette indépendance, associée à la sphère publique $\left.^{1}\right)$. Cette transformation de l'intimité se calque sur la transformation des individus eux-mêmes ${ }^{2}$. Avant d'être membres du couple, hommes et femmes se définissent d'abord comme individus. Après avoir été première, la communauté cède la place à ce que je nomme «l'individu individualisé». Il s'agit d'un individu dont la définition ne dépend pas avant tout de son appartenance à tel ou tel groupe, y compris son appartenance à son couple, dont l'identité ne peut se construire que par un mouvement d'émancipation, par une certaine désaffiliation positive (De Singly, 2003). On assiste à un processus général de renforcement progressif des individus face au groupe d'appartenance, du droit des hommes et femmes, et des enfants également, à décider d'euxmêmes et à rester eux-mêmes propriétaires (notamment de leur corps). Par voie de conséquence, ces hommes et ces femmes sont plus sensibles à ce que la vie commune leur coûte. Ils veillent à ce que leur épanouissement et leur liberté individuelle ne soient pas trop sacrifiés. Vis-à-vis de leur conjoint, ils veulent préserver des territoires personnels, une certaine intimité personnelle 3 . Pour eux, la vie commune peut être source d'empiètements, aussi peuvent-ils lutter contre la «fusion» avec l'autre. Aussi cherchent-ils à inventer des formes de vie conjugale qui sachent respecter leur revendication de rester libres tout en devenant des individus «avec».

\section{Le modèle du primat de l'intimité personnelle}

Pour Anthony Giddens, à cette nouvelle période de l'intimité correspond un idéaltype de relation, la «relation pure $»^{4}$. Il s'agit d'une relation sexuellement et émotionnellement égalitaires, au sein de laquelle compte avant tout l'autonomie individuelle, la qualité des échanges, l'intensité émotionnelle. Elle se caractérise aussi par le fait que la rupture est inscrite comme horizon possible dès le début. Elle est «autoréférentielle en ce sens qu'elle dépend fondamentalement de la satisfaction et des récompenses qui

1. Pour U. Beck (1992), il s'agit du premier processus d'individualisation.

2. On peut relire Norbert Elias (1973) dans cette optique.

3. Est-ce que la vie sexuelle est la dimension la plus représentative de l'intimité personnelle? Cela semble évident à beaucoup (pour exemple, un numéro d'une revue Mana (1997) qui, sous le titre «Approches sociologiques de l'intime», donne une grande place à la sexualité. Ce postulat mérite d'être questionné, le récit de Catherine Millet (2001) pouvant servir comme exemple: la sexualité peut aussi ne pas être vécue comme le territoire le plus intime. Le présent article se situe dans une perspective selon laquelle l'intimité personnelle se construit dans le secret (Simmel, 1999). Cf. Finkenauer et Hazam (2000) sur ce point et aussi Gagnon (1993).

4. Ce modèle a été critiqué en Grande-Bretagne notamment, selon une perspective différente de celle développée dans cet article. Pour L. Jamieson (1999), c'est l'écart entre le modèle et la réalité des rapports entre les genres qui pose problème et qui autorise à s'interroger sur les fonctions, à la suite de Morgan (1991, 1996), de tels modèles.

5. L'égalité entre les genres ne sera pas traitée dans cet article. Si l'égalité au plan du pouvoir a augmenté dans le couple, celle au plan du partage du travail domestique progresse peu. Cf. pour la France, De Singly $(1987,2001 b)$. 
résultent de la relation elle-même» (Giddens, 1993, p. 465-466; 1991, p. 244). Ce n'est donc pas une relation instituée qui échapperait à la maîtrise des individus qui l'ont créée. Si cette relation se poursuit, c'est uniquement en raison des bilans positifs, régulièrement établis par les partenaires.

Le terme «pur» renvoie à la rupture historique avec des relations qui dépendent des devoirs sociaux, des contraintes de la parenté, des obligations traditionnelles; il désigne le fait que cette relation ne s'inscrit dans aucun autre critère sinon la relation elle-même (Giddens, 1992, p. 63). Cette relation a surtout, semble-t-il, un but instrumental, celui de procurer à l'individu les conditions idéales de la construction de soi, de son épanouissement personnel. Autrement dit, la relation semble importer plus que la personne aimée. La relation pure et sa durée incertaine participent non seulement de ce que l'auteur nomme une «separating and divorcing society», mais aussi de la société du risque: le futur est devenu incertain également dans la sphère privée.

La relation pure repose sur ce que Giddens nomme l'amour confluent qui s'oppose à l'amour romantique — défini, lui, par le «toujours», par une inégalité entre les genres, et par une demande d'exclusivité sexuelle. Toujours selon Giddens, la relation pure se distingue de la relation compulsive au sein de laquelle l'individu est pris dans des liens de dépendance et de fusion, rendant plus difficile l'autonomie personnelle. L'individu moderne, devant exister par lui-même, ne doit ni dépendre de cet autrui, ni se confondre avec lui. L'amour confluent est un amour lucide où chacun, restant à sa place, n'est pas tenté par la perte de soi dans l'échange des serments et des mots d'amour. Dans ce modèle, la femme ou l'homme parvient à se préserver, à éviter ce qu'il considère comme des empiètements. C'est le rêve d'une vie commune où les partenaires sont «ensemble sans se confondre» : ils sont avant tout amis, autorisés à pratiquer un "amour ouvert, c'est-à-dire non exclusif, qui respecte et favorise l'épanouissement de chacun» (Chaumier, 1999, p. 215). Chacun se protège afin de pouvoir «vivre une vie sociale indépendante de [son] conjoint» (p. 214).

Ce modèle de la «relation pure» est considéré, selon nous, comme celui de la suprématie de l'intimité personnelle sur l'intimité conjugale. Il s'oppose au modèle de la fusion, caractérisé par la suprématie de l'intimité conjugale sur l'intimité personnelle. D’un côté le primat des individus individualisés, de l'autre, le primat du couple.

\section{Un autre modèle, respectant un équilibre entre l'intimité personnelle et l'intimité conjugale}

Selon nous, le modèle de la «relation pure», mis en forme par Giddens, ne résout pas tous les problèmes posés par la modernité. En effet, on ne comprend pas bien la nature de ce qui est «commun» entre les deux partenaires: tout se passe comme si le principal accord entre eux portait sur le fait de ne pas être trop liés ${ }^{6}$, de ne pas être dépendants,

6. «La caractéristique essentielle commune aux mariages et aux relations sexuellement ouverts est l'engagement mutuel de ne pas se montrer sexuellement exclusif» (Knapp, Whitehurst, 1981, p. 56, cité par Chaumier, 1999, p. 216). 
d'échapper à la fusion aliénante. Dans la définition de la relation pure, on appréhende bien les raisons qui conduisent les partenaires à refuser l'excès de partage de temps commun, pouvant mener à une confusion identitaire et à un lien de dépendance, mais on appréhende moins la manière dont se construit une communauté minimale autorisant cette relation à apparaître comme méritant d'être nommée «couple», ou «vie à deux». Est-il possible d'avoir des échanges avec l'autre, qui sous-tendent le projet réflexif de l'un et de l'autre, sans en même temps faire aussi des choses ensemble? De telles pratiques communes qui ne sont pas exclues du modèle sont peu commentées par Giddens, à l'exception de la sexualité (qui peut être conjugale ou non). On ne comprend pas bien comment se crée la «confiance mutuelle entre partenaires, étroitement associée à l'accomplissement de l'intimité, c'est-à-dire pour l'essentiel, à la connaissance de l'autre et à la connaissance de soi» (Giddens, 1993, p. 466).

Ce modèle nous semble sous-estimer certaines demandes des individus modernes: leur besoin de sécurité ontologique (De Singly et Charland, 2002) qui peut s'inscrire sous les formes de la fidélité et de l'engagement à long terme; la demande de parentalité; et enfin les satisfactions tirées de la vie commune. Il faut appréhender les manières dont les uns et les autres parviennent à trouver un certain équilibre entre l'intimité personnelle et l'intimité conjugale, entre l'indépendance personnelle et la demande de réunion conjugale, sans les référer uniquement à un seul modèle. En effet, Giddens oublie ce qu'Erving Goffman souligne dans son analyse des relations: un même fait peut être perçu comme une invasion (demandant un rituel de réparation) ou comme la preuve, au contraire, de l'existence d'un lien, le signe d'un rituel de confirmation. Tout se passe comme si, dans le modèle de la relation pure, l'exclusivité sexuelle, par exemple, ou encore le fait de faire telle ou telle activité ensemble, était nécessairement une infraction à l'indépendance des conjoints. La «fusion» est toujours perçue, dans la perspective de Giddens, du point de vue de l'invasion possible. Cet auteur oublie trop l'autre face de toute relation: la possibilité de créer, de maintenir une relation grâce à un renoncement volontaire d'un de ses territoires personnels. C'est ainsi que la relation sexuelle peut être atteinte: invasion ou, au contraire, symbole positif de la relation et signe de l'amour? Pourquoi penser que les individus individualisés ne seraient surtout sensibles qu'à la préservation d'un soi indépendant, ne rêveraient que de l'absence de tout empiètement? C'est oublier l'attraction de ce que nous nommons «l'individualisme relationnel», c'est-à-dire la possibilité de se construire comme une identité grâce à une relation avec un autrui significatif. On peut ainsi dessiner un autre modèle de relation au sein des sociétés modernes avancées: un modèle qui ne rejetterait ni la fusion — plutôt caractéristique de la période antérieure — ni l'autonomie. Il sera désigné sous le terme de «double respect», celui de l'individu individualisé et celui de la communauté partielle, celui de la recherche d'un équilibre entre l'intimité personnelle et l'intimité conjugale. 


\section{QUESTIONS DE MÉTHODE}

Pour appréhender plus complètement ces modèles — au sens d'idéal-type —, on présentera les résultats d'une enquête quantitative par questionnaire passée auprès de 828 individus, hommes et femmes, âgés entre 30 et 50 ans, vivant en couple et sous le même toit depuis plus d'un an. Ils habitent la région parisienne. Ils devaient avoir au moins un niveau scolaire correspondant à l'achèvement des études secondaires (sanctionnées en France par un diplôme, le baccalauréat). Et les individus interrogés devaient avoir un portable depuis plus de six mois. Cette enquête est donc limitée culturellement afin de diminuer les oppositions sociales et de mettre mieux en lumière les variations internes de formes de vie privée. Le choix de prendre des individus au moins bacheliers repose sur le fait que la théorie de Giddens s'applique nettement plus, au moins avant une diffusion sociale, aux individus les mieux dotés scolairement. Il ne s'agit donc pas de réaliser une enquête représentative de la vie de couple en France ou dans la région parisienne. Il s'agit de comprendre comment le processus d'individualisation se mêle à la construction d'une vie commune à partir de l'appropriation d'un équipement considéré socialement comme «personnel» et d'autres dimensions des relations entre les partenaires ${ }^{7}$. Les enquêtes plus représentatives sont réalisées, en France, dans des cadres qui n'autorisent pas le point de vue théorique. La présente enquête se situe à un autre niveau, moins descriptif. Ce qu'elle cherche avant tout, ce sont des « relations de relations». Les données sont fournies surtout comme indicateurs de l'existence de processus différenciés. D'une certaine façon cette enquête relève d'une logique qualitative plus centrée sur le «comment» que sur le «pourquoi», sur le processus que sur les déterminants sociaux. Elle repose donc sur un pari méthodologique que les idéauxtypes peuvent être illustrés non seulement, comme c'est le plus fréquemment le cas, par des récits, des entretiens, mais aussi par des données quantitatives. L'intérêt de cet usage du raisonnement quantitatif est de faire découvrir des associations entre variables non prévues et d'engendrer ainsi de nouveaux questionnements théoriques (Passeron, 1991).

Les trois groupes de référence-correspondant aux trois modèles — ont été élaborés à partir des réponses à deux questions. Les personnes interrogées devaient indiquer si elles étaient «tout à fait d'accord, assez d'accord, pas vraiment d'accord, pas du tout d'accord» avec l'affirmation selon laquelle «vivre en couple c'est accepter que chacun ait une vie autonome». Des individus interrogés, $77 \%$ déclarent être d'accord avec cette affirmation. Cela démontre la force de la valeur de l'indépendance au sein de la vie conjugale à l'époque contemporaine. Mais à l'autre question qui demandait si «vivre en

7. L'enquête (entretiens exploratoires, rédaction du questionnaire, passation des questionnaires) a été réalisée pendant le premier semestre de 2000, dans le cadre d'un enseignement de méthodologie sociologique, à la faculté de sciences humaines et sociales de la Sorbonne, Université de Paris V. Les questionnaires, comportant environ 150 questions, ont été passés en mars-avril 2000. Sous ma direction, l'équipe d'enseignants-chercheurs était composée de Vincenzo Cicchelli, Catherine Cicchelli-Pugeault, Christophe Giraud et Olivier Martin. Que ceux-ci, et tous les étudiants qu'ils ont encadrés, soient remerciés. Une première exploitation de l'enquête a été effectuée, plus centrée sur le téléphone portable: cf. Martin et De Singly (2002). 
couple suppose de tout partager ", $44 \%$ des individus sont également d'accord avec ce second énoncé. C'est le signe que pour certains, les deux principes de fusion et d'autonomie sont compatibles et donc qu'il existe un autre choix que celui de la relation pure, même pour ceux et celles qui valident le processus d'individualisation.

Le croisement entre les distributions pour les deux questions permet d'approcher la diversité des façons de pondérer individualisation et «communauté». Si 39\% des individus (323 personnes) approuvent l'autonomie des individus et critiquent la fusion - ce groupe, proche du modèle de la relation pure, est celui de la défense de l'intimité personnelle — un autre groupe est presque aussi important: $37 \%$ des individus (soit 306 personnes) approuvent l'autonomie des individus et également le fait de tout partager dans le couple. C'est le groupe du double respect qui correspond à la recherche d'un équilibre entre la communauté et l'indépendance. Un troisième groupe - nettement plus petit, $16 \%$ des individus, soit 134 personnes - est favorable au primat de l'intimité conjugale, à la fusion ${ }^{9}$ tout en désapprouvant l'autonomie. Il sera nommé modèle de l'intimité conjugale, ou celui de la communauté.

\section{Le primat de l'intimité personnelle, celui de l'intimité conjugale et le refus de les hiérarchiser}

\section{Quelques territoires personnels}

Les trois groupes se différencient à propos de la première dimension de l'intimité personnelle: la possibilité d'avoir des territoires personnels, protégés de l'invasion par le conjoint, approchés par le respect du courrier personnel reçu à la maison, par l'individualisation du téléphone portable (les indicateurs étant que le conjoint s'autorise ou non de répondre à la place du répondant sur le téléphone de ce dernier, que le conjoint connaisse ou non le code PIN $^{10}$ du portable, par le fait d'avoir programmé des numéros de téléphone inconnus du conjoint). Dans la perspective de Goffman, ces questions peuvent servir d'indicateur aux réserves d'information — «ensemble de faits qui concernent l'individu et dont ce dernier entend contrôler l'accès» (Goffman, 1973, p. 52) - et aux domaines réservés de la conversation — «le droit qu'a l'individu d'exercer un certain contrôle sur qui peut lui adresser la parole et quand» (p. 53).

Dans tous les cas, l'ordre théorique est respecté: les individus classés dans le groupe de la relation pure défendent plus que les partisans de la communauté leurs réserves d'information et leurs domaines réservés de la communication. Et les individus qui

8. Ce groupe est nettement plus nombreux que celui des partisans de la fusion. Cela contredit la thèse de Kalmijn et Bernasco (2001) qui estiment, à partir de leur enquête aux Pays-Bas, que «les couples contemporains ne peuvent pas être caractérisés comme hautement individualisés ». On remarquera notamment que l'enquête de Kalmijn et Bernasco n'approche pas l'intimité personnelle par le secret, ils la mesurent surtout par des questions portant sur des activités de loisirs.

9. Un quatrième groupe, nettement moins nombreux ( $7 \%$, soit 58 individus), refuse la fusion conjugale et l'autonomie individuelle. Il ne sera pas étudié dans cet article.

10. C'est le code d'entrée personnel pour pouvoir utiliser le téléphone. 
TABLEA U

Quelques réserves d'information et quelques domaines réservés de la conversation (\% horizontaux)

\begin{tabular}{|c|c|c|c|}
\hline \multicolumn{4}{|c|}{ 1. LE COURRIER PERSONNEL OUVERT PAR LE CONJOINT } \\
\hline & SOUVENT & DE TEMPS EN TEMPS & JAMAIS \\
\hline Double respect & 17,3 & 34,1 & 48,6 \\
\hline Intimité personnelle & 4,9 & 17,6 & 77,1 \\
\hline Intimité conjugale & 30,0 & 31,6 & 39,1 \\
\hline \multicolumn{4}{|c|}{ 2. LE CONJOINT RÉPOND À LA PLACE DE L'INDIVIDU QUESTIONNÉ QUAND LE PORTABLE DE CE DERNIER SONNE } \\
\hline & \multicolumn{2}{|c|}{ oul } & NON \\
\hline Double respect & \multicolumn{2}{|c|}{52,0} & 48,0 \\
\hline Intimité personnelle & \multicolumn{2}{|c|}{33,4} & 66,6 \\
\hline Intimité conjugale & \multicolumn{2}{|c|}{56,0} & 44,0 \\
\hline \multicolumn{4}{|c|}{ 3. LA GÊNE OU NON PENDANT LA TOILETTE PROVOQUÉE PAR LA PRÉSENCE DU CONJOINT } \\
\hline & \multicolumn{2}{|c|}{ DÉRANGE } & NE DÉRANGE PAS \\
\hline Double respect & \multicolumn{2}{|c|}{13,3} & 86,7 \\
\hline Intimité personnelle & \multicolumn{2}{|c|}{21,3} & 78,7 \\
\hline Intimité conjugale & \multicolumn{2}{|c|}{7,5} & 92,5 \\
\hline \multicolumn{4}{|c|}{ 4. LA FEM ME A GARDÉ SON NOM } \\
\hline & $\begin{array}{l}\text { OUI, DANS LE } \\
\text { CONCUBINAG }\end{array}$ & OUI, DANS LE MARIAGE & NON, ELLE L'A PERDU \\
\hline Double respect & 37,0 & 6,7 & 56,3 \\
\hline Intimité personnelle & 46,1 & 12,5 & 41,4 \\
\hline Intimité conjugale & 28,8 & 12,1 & 59,1 \\
\hline
\end{tabular}

recherchent le double respect se situent entre ces deux groupes. Les personnes favorables à la communauté estiment que leur identité personnelle se confond davantage avec celle de leur partenaire. Ce positionnement des trois groupes s'observe aussi avec deux autres indicateurs: le fait de garder ou non son nom après le mariage (ce dernier est un usage encore peu fréquent pour les femmes en France) et le fait d'être gêné par la présence du conjoint dans la salle de bains pendant la toilette (tableau 1).

L'amplitude de la protection de soi varie selon les questions, elle permet de savoir ce que le conjoint peut se permettre ou non. La question obtenant le moins de réponses indiquant la possibilité d'une fermeture de soi vis-à-vis de son partenaire est celle portant sur la toilette. Déclarer être gêné par la présence de l'autre dans la salle de bains revient à penser qu'il s'agit presque d'une intrusion. Cela est refusé par la très grande majorité des hommes et des femmes. Le «corps», qui est une propriété per- 
sonnelle, doit, malgré tout, être partagé. Ne pas vouloir être vu pendant sa toilette est perçu comme une revendication trop élevée d'intimité personnelle. Il existe un désaccord sur le patronyme entre les partisans de l'intimité conjugale et ceux de l'intimité personnelle. Avoir un seul nom pour la famille peut être codé de deux manières différentes: pour le groupe de la relation pure, c'est une perte, pour la femme, de son identité alors que pour le groupe de la communauté, c'est le symbole même de l'union, de l'existence d'une équipe conjugale. Le nom, commun ou non, traduit un rapport à l'engagement.

Le groupe du double respect se rapproche plus de la relation pure pour la question du nom. Pour les réactions face au conjoint qui ouvre le courrier ou qui répond à la place de soi, ce groupe est plus près de celui de la communauté. Pour les autres questions, il se situe entre les deux ${ }^{11}$. Le compromis élaboré par ceux et celles qui refusent le primat d'une intimité sur une autre differe donc. Ces partisans insistent plus sur leur identité personnelle, sur le nom. Ils estiment que le courrier peut être mis en commun, sans doute comme marque de confiance. Ils n'ont rien à cacher, voulant rester eux-mêmes - par le nom notamment — tout en démontrant leur appartenance conjugale par le refus du secret, notamment du courrier ou des coups de fil.

Les différences entre les groupes s'estompent à propos de la conversation conjugale, abordée par les questions: «Est-ce que l'on raconte spontanément sa journée à son conjoint? Attend-on que le partenaire demande? Adopte-t-on plutôt une attitude silencieuse?» Les partisans de la communauté ne valorisent pas plus que les partisans de la relation pure la conversation conjugale. Ce résultat est très important puisque, dans la perspective ouverte par Peter Berger (1988), le critère le plus important de la vie conjugale ne distingue pas significativement les groupes. C'est en effet dans la conversation que les partenaires élaborent progressivement un monde commun qui soutient leurs identités respectives. La séparation des pratiques et la protection de soi n'excluent pas le partage par le biais des échanges verbaux. Ces conversations sont une ouverture réciproque. Centrée davantage sur la parole que sur les activités partagées, la relation pure ne valorise donc pas que certains éléments de la vie commune.

\section{LE CHOIX DU COMMUN OU DU PERSON NEL}

Le droit à avoir des activités sans le conjoint est, en effet, très nettement, revendiqué par les partisans de l'intimité personnelle. Les deux dernières fois ${ }^{12}$ où ils ont acheté des vêtements, où ils sont allés voir leurs parents, où ils sont partis en vacances, où ils sont sortis, ils l'ont fait assez souvent sans être accompagnés de leur partenaire (tableau 2). Là encore, cela ne signifie pas le rejet du collectif. Ainsi, c'est une minorité des partisans de la relation pure qui sont sortis les deux dernières fois sans leur conjoint, ils sont net-

11. On calcule la différence entre le double respect et la relation pure, d'une part, et entre le double respect et la communauté, d'autre part.

12. Chaque question était répétée deux fois: la dernière fois...; l'avant-dernière fois... À partir des réponses, on construisait un score. 
TABLEA U 2

Les pratiques communes ou séparées (\% horizontaux)

\begin{tabular}{|c|c|c|c|}
\hline \multicolumn{4}{|c|}{ 1. ACHETER DES VÊTEMENTS AVEC SON CONJOINT LES DEUX DERNIÈRES FOIS } \\
\hline & AUCUNE FOIS & UNE FOIS & DEUX FOIS \\
\hline Double respect & 41,2 & 27,6 & 31,3 \\
\hline Intimité personnelle & 60,5 & 24,2 & 15,3 \\
\hline Intimité conjugale & 39,1 & 22,6 & 41,3 \\
\hline \multicolumn{4}{|c|}{ 2. ALLER EN VACANCES SANS SON CONJOINT LES DEUX DERNIÈRES FOIS } \\
\hline & AUCUNE FOIS & UNE FOIS & DEUX FOIS \\
\hline Double respect & 55,8 & 16,6 & 27,6 \\
\hline Intimité personnelle & 30,7 & 20,5 & 48,8 \\
\hline Intimité conjugale & 65,2 & 17,4 & 17,4 \\
\hline \multicolumn{4}{|c|}{ 3. ALLER VOIR SES PARENTS SANS SON CONJOINT LES DEUX DERNIÈRES FOIS } \\
\hline & DEUX FOIS & UNE FOIS & AUCUNE \\
\hline Double respect & 47,6 & 33,1 & 19,3 \\
\hline Intimité personnelle & 34,1 & 44,6 & 21,2 \\
\hline Intimité conjugale & 60,7 & 22,1 & 17,2 \\
\hline \multicolumn{4}{|c|}{ 4. SORTIR SANS SON CONJOINT LES DEUX DERNIÈRES FOIS } \\
\hline & DEUX FOIS & UNE FOIS & AUCUNE \\
\hline Double respect & 9,0 & 27,2 & 63,8 \\
\hline Intimité personnelle & 13,4 & 45,1 & 16,4 \\
\hline Intimité conjugale & 4,5 & 16,4 & 79,1 \\
\hline \multicolumn{4}{|c|}{ 5. NOMBRE D'ACTIVITÉS SANS LE CONJOINT } \\
\hline & AUCUNE & UNE & DEUX ET PLUS \\
\hline Double respect & 31,6 & 35,6 & 32,8 \\
\hline Intimité personnelle & 18,6 & 29,7 & 51,6 \\
\hline Intimité conjugale & 39,6 & 37,3 & 23,1 \\
\hline \multicolumn{4}{|c|}{ 6. NOMBRE D'ACTIVITÉS AVEC LE CONJOINT } \\
\hline & AUCUNE & UNE & DEUX ET PLUS \\
\hline Double respect & 23,8 & Une & 49,8 \\
\hline Intimité personnelle & 17,3 & 26,3 & 54,6 \\
\hline Intimité conjugale & 26,1 & 28,1 & 55,2 \\
\hline
\end{tabular}


tement plus nombreux à être sortis une fois seul et une fois ensemble ( $45 \%$ contre $13 \%)$.

Le groupe de la relation pure sort plus que les autres, alternant activités communes et activités personnelles. Ce résultat permet de comprendre qu'il ne faut pas confondre le droit de sortir sans son conjoint avec le contenu réel des pratiques ${ }^{13}$. Ce droit est affirmé comme une revendication, il ne signifie pas pour autant: «Je veux sortir toujours seul.» La différence entre les trois groupes apparaît nettement avec le rapport entre le poids des pratiques communes fréquentes et celui des pratiques personnelles fréquentes: le collectif prend plus ou moins de place. Alors que pour la relation pure, il ne pèse pas plus que les pratiques individuelles, pour les individus qui recherchent un équilibre, le commun l'emporte. En reprenant les quatre questions portant sur les deux dernières fois, on observe que le groupe du double respect est plus proche des communautaires pour les vêtements et pour les vacances et plus proche de la relation pure pour les sorties et pour les visites aux parents. Ils retiennent donc certains éléments du modèle de la fusion et d'autres du modèle de l'autonomie. C'est ainsi qu'ils prennent plus que les deux autres groupes leur petit déjeuner ensemble: $42 \%$ contre respectivement $33 \%$ pour la relation pure et $31 \%$ pour la communauté.

Les questions sur les vacances comportaient une distinction dans les réponses: «Oui, vous avez pris au moins quatre jours de vacances sans votre conjoint parce que vous n’aviez pas toujours les mêmes congés», «Oui... et pourtant vous avez eu les mêmes congés». Dans le premier cas, l'individu partait, en pouvant avoir l'excuse du décalage des rythmes; dans le second, l'individu revendiquait explicitement par son acte le fait de prendre quelques jours seul. La revendication du droit aux vacances seul est aussi souvent énoncée par les partisans du double respect que par ceux de la relation pure. C'est une preuve de plus que les premiers oscillent entre deux logiques: celle d'être avec le conjoint et celle de pouvoir être seul, adoptant l'une ou l'autre selon les circonstances.

On peut alors s'étonner que pour l'argent, ce groupe ne prenne pas plus que les autres groupes la solution de compromis, en ayant à la fois un compte personnel bancaire et un compte commun ${ }^{14}$. Aujourd'hui, l'argent est géré séparément, au moins pour une grande part puisqu'un quart, seulement, des partisans de l'intimité conjugale ont exclusivement un compte conjoint. Les différences observées sont peu significatives même si le groupe de la relation pure possède un peu plus que les autres des comptes séparés sans compte conjoint. Pourquoi cet élément si important est-il devenu non commun, des enquêtes devraient-elles aborder ce fait? Pour la carte de crédit, associée au compte, le groupe du double respect adopte une ligne communautaire puisque leur conjoint en connaît le code secret. Ils prennent sans doute cette connaissance comme un signe de confiance. La difficulté propre à l'établissement d'une rela-

13. C'est le défaut du travail centré uniquement sur les représentations, comme ceux de Kellerhals et al. (1982) et de De Singly (1987), qui perçoivent surtout les normes de la conduite et non les arrangements de la vie quotidienne.

14. Soit: $42 \%$ des partisans de l'intimité personnelle, $44 \%$ des partisans de l'intimité conjugale et $38 \%$ des partisans du double respect. 
tion dans les sociétés contemporaines privilégiant un individu «indépendant et autonome» (Renaut, 1989) est de faire en sorte que l'ouverture à autrui reste possible sans devenir une intrusion. Les hommes et les femmes se trouvent toujours dans une situation ambiguë puisque la définition des ouvertures possibles sans atteinte dépend pour une grande part de leur interprétation. Chacun doit définir ce qu'il considère a) comme «personnel» strictement et ne devant pas être partagé, b) comme "personnel» et pouvant être partagé, et c) ce qui est peu personnel. L'argent relève surtout de la seconde catégorie, les amis aussi. Très majoritairement l'individu, même en couple, peut avoir au moins quelques amis non partagés avec son conjoint. Même les partisans de l'intimité conjugale acceptent la création et le maintien de relations avec d'autres proches, hors du régime de la communauté. Le clivage se fait donc non entre l'intimité conjugale et son refus, mais selon l'intensité de l'ouverture autorisée, c'est-à-dire selon le nombre d'amis personnels. Même si le compromis est plus fort chez les individus du groupe «double respect», il est mis en ouvre par l'ensemble des hommes et des femmes. La position du «tout fusion» ou «tout individuel» n'est pas possible, étant donné la force de la norme du respect de l'individu, d'une part, et la nécessité de pratiques confirmant l'existence d'une relation, d'autre part.

\section{LE RAPPORT À L'ENGAGEMENT MATRIMONIAL}

Dans Mariages au quotidien (1982) — une recherche sur les représentations des échanges entre les partenaires - Jean Kellerhals et son équipe ont montré, en faisant varier dans des scénarios la durée du couple, comment cette dimension du temps affectait le degré de fusion conjugale souhaité. Plus la durée était limitée, plus l'indépendance primait. Dans le cadre du modèle de la relation pure, Giddens insiste, lui aussi, fortement sur les formes de l'engagement. Avec d'autres (Roussel, 1989; De Singly, 1987, 1996), il énonce les raisons du déclin de l'institution du mariage et de la montée de l'union libre (ou du concubinage): des hommes et des femmes ne veulent pas que cette institution les enferme dans des rôles qu'ils ne voudraient plus tenir lorsque les satisfactions tirées du mariage ne seront plus suffisantes. Ils souhaitent que leur engagement reste personnel pour que leur identité ne soit pas masquée par des rôles conjugaux et familiaux. Le doute vis-à-vis de l'utilité du mariage va de pair avec le fait de ne pas exclure de sa propre union la possibilité de rompre. Ce sont deux attitudes qui expriment la même revendication de «rester soi-même».

Cette revendication est très forte chez les partisans de l'intimité personnelle. Moins de la moitié des membres de ce groupe déclarent que l'engagement à long terme leur plaît. Ils sont, en revanche, plus nombreux que les deux autres groupes à ne pas exclure une séparation ou un divorce. Ils ne rêvent pas du divorce, mais ils ne veulent pas rester à tout prix, peu importe la relation, avec quelqu'un qui ne serait plus proche affectivement. La relation conjugale ne doit plus être gouvernée par les institutions; elle doit rester «libre». Ces partisans de l'intimité personnelle sont en grand désaccord avec l'affirmation selon laquelle le mariage est une nécessité pour vivre à deux ( $72 \%$ ne sont pas d'accord, contre $39 \%$ chez les partisans du double respect et $28 \%$ chez 
TA B LEA U 3

L'engagement conjugal (\% horizontaux)

\begin{tabular}{|c|c|c|c|c|c|c|}
\hline \multicolumn{7}{|c|}{ Le rapport au mariage } \\
\hline \multicolumn{7}{|c|}{ 1. LE SENS DE SON UNION CONJUGALE } \\
\hline & \multicolumn{2}{|c|}{$\begin{array}{l}\text { UNION QUI PEUT DURER } \\
\text { MAIS DISSOLUBLE }\end{array}$} & \multicolumn{2}{|c|}{$\begin{array}{l}\text { C'EST POUR LA VIE MAIS } \\
\text { SÉPARATION POSSIBLE SI } \\
\text { MOTIFS SÉRIEUX }\end{array}$} & \multicolumn{2}{|c|}{$\begin{array}{c}\text { C'EST POUR LA VIE ET } \\
\text { SÉPARATION TRÈS DIFFICILE } \\
\text { À ENVISAGER }\end{array}$} \\
\hline Double respect & \multicolumn{2}{|l|}{9,9} & \multicolumn{2}{|c|}{42,2} & \multicolumn{2}{|r|}{47,8} \\
\hline Intimité personnelle & \multicolumn{2}{|l|}{29,7} & \multicolumn{2}{|c|}{46,8} & & 24,4 \\
\hline Intimité conjugale & \multicolumn{2}{|l|}{5,3} & \multicolumn{2}{|c|}{35,1} & & 59,6 \\
\hline \multicolumn{7}{|c|}{ 2. A DÉJÀ VÉCU UNE SÉPARATION OU UN DIVORCE } \\
\hline & \multicolumn{3}{|c|}{ OUI } & \multicolumn{3}{|c|}{ NON } \\
\hline Double respect & \multicolumn{3}{|c|}{31,3} & \multicolumn{3}{|c|}{68,7} \\
\hline Intimité personnelle & \multicolumn{3}{|c|}{43,3} & \multicolumn{3}{|c|}{56,7} \\
\hline Intimité conjugale & \multicolumn{3}{|c|}{29,1} & \multicolumn{3}{|c|}{70,9} \\
\hline \multicolumn{7}{|c|}{ Le sens de la vie commune } \\
\hline \multicolumn{7}{|c|}{ 1. LES DEUX PRIORITÉS DANS SA VIE } \\
\hline & LE COUPLE & & ENFANTS & LA PROFESS & SION & LE TEMPS LIBRE \\
\hline Double respect & 74,3 & & 61,9 & 29,4 & & 24,1 \\
\hline Intimité personnelle & 67,3 & & 52,9 & 32,3 & & 34,3 \\
\hline Intimité conjugale & 76,8 & & 72,4 & 30,5 & & 11,2 \\
\hline \multicolumn{7}{|c|}{ 2. VIVRE À DEUX C'EST SURTOUT } \\
\hline & $\begin{array}{c}\text { VIVRE } \\
\text { UNE PASSION }\end{array}$ & AVOI & $\begin{array}{l}\text { DES PROJETS } \\
\text { DMMUNS }\end{array}$ & $\begin{array}{r}\text { COMPTER SU } \\
\text { PROCHE }\end{array}$ & $\begin{array}{l}\text { UR UN } \\
\text { E }\end{array}$ & $\begin{array}{l}\text { FONDER UNE } \\
\text { FAMILLE }\end{array}$ \\
\hline Double respect & 26,0 & & 29,7 & 5,6 & & 38,7 \\
\hline Intimité personnelle & 21,1 & & 43,2 & 12,9 & & 22,8 \\
\hline Intimité conjugale & 18,6 & & 35,8 & 2,2 & & 43,3 \\
\hline
\end{tabular}

les partisans de l'intimité conjugale). Le mariage devient un événement "privé», facultatif, qui ne regarde que les personnes concernées.

Sur l'engagement à long terme, sur le mariage, sur le divorce, le groupe du double respect élabore un équilibre qui ne se situe pas entre les deux principes. Il opte plutôt pour l'intimité conjugale puisque ses réponses ressemblent à celles des communautaires. En revanche, sur les questions du Pacte civil de solidarité, concernant les couples homosexuels et hétérosexuels, et sur l'homosexualité perçue comme «forme acceptable de sexualité», l'équilibre est recherché de l'autre côté, avec le groupe de l'intimité personnelle. Les partisans de la double intimité privilégient pour eux une durée associée à l'institution du mariage, sans que cela leur interdise de penser que d'autres peu- 
vent faire des choix différents. Ils expriment une certaine approbation des principes proches de la relation pure mais ils n'y adhèrent pas à titre personnel. Si cela est vrai, cela éclairerait la manière dont ils résolvent la tension entre les deux intimités: ces hommes et femmes adopteraient, pour leur vie privée, plutôt une ligne de conduite calquée sur celle des communautaires, tout en estimant que dans les sociétés contemporaines, il peut exister d'autres façons de construire son existence (tableau 3).

\section{LA QUESTION DE L'ENFANT}

Dans l'élaboration de son modèle, Giddens omet de préciser la place de l'enfant qui, par sa présence, modifie le sens du groupe conjugal_comme le soulignait déjà Durkheim (1906). Le fait que les partenaires deviennent aussi parents engendre un lien supplémentaire entre eux - que d'ailleurs la législation sur le divorce en France estime indissociable puisque l'autorité parentale reste conjointe même après la séparation. On peut de plus penser que la décision de faire un enfant est un signe d'un certain engagement temporel. À la question sur les deux priorités dans la vie, l'enfant et le fait de s'en occuper occupent une place décroissante en passant du groupe de l'intimité conjugale à celui du double respect, puis à celui de l'intimité personnelle. Autrement dit, l'intimité conjugale est associée à un attachement à l'intimité familiale. On le perçoit aussi dans le fait que, pour une question sur le sens de la «vie à deux», l'expression de «fonder une famille»— qui désigne la naissance des enfants — est plus retenue par les communautaires (mais le groupe du double respect en est proche).

Le nombre d'enfants de chacun révèle aussi, en actes, cet engagement, variable, dans la famille: $34 \%$ des partisans de l'intimité conjugale, $15 \%$ de ceux de l'intimité personnelle et $18 \%$ de ceux de la double intimité ont trois enfants et plus. Inversement, $30 \%$ des groupes de la double intimité et de l'intimité personnelle, contre $18 \% \mathrm{du}$ groupe de la fusion, sont sans enfant ${ }^{15}$. L'intimité conjugale est peu compatible avec l'absence d'enfants. Elle s'inscrit dans la famille de deux ou trois enfants. La «famille nombreuse» (qui en France, juridiquement, commence à trois) est deux fois plus importante dans ce groupe que dans les deux autres. Là, les partisans du double respect sont plus proches des partisans de l'intimité personnelle. Ils n'insistent pas, selon leurs réponses dans les représentations et dans leurs conduites, avec la même intensité sur le fait d'avoir des enfants. Sans doute hésitent-ils entre une conception plus «conjugale» de l'intimité et une vision plus «familiale», alors que les communautaires n'envisagent pas le conjugal sans le familial.

À la question sur les priorités dans la vie, ceux qui prônent l'intimité personnelle retiennent, plus que les autres, la réponse «Avoir du temps libre». Ce terme est alors compris dans un sens plus spécifique que le sens habituel (temps hors travail et hors contrainte): le temps hors famille. C'est le temps qui reste pour soi, pour ses relations avec ses amis, pour sortir. On peut s'étonner que la réponse «Réussir sa vie professionnelle» ne varie pas selon les groupes. On s'attendait à ce que la réalisation de soi par

15. Il n'y a pas de différence significative d'âge entre les trois groupes. 
la carrière caractérise le groupe de la relation pure, ce qui n'est pas le cas. Il faut aussi remarquer deux réponses de ce groupe à la question sur la définition de la vie à deux: les partisans de l'intimité personnelle ne valorisent pas plus que les autres la passionqui s'oppose souvent dans l'imaginaire à la routine des habitudes et des rôles conjugaux. Cette faible adhésion s'explique, peut-être, parce que la passion augmente la dépendance, non pas objective, mais relationnelle et donc s'oppose à la liberté de l'individu, même au sein d'une relation élective. Ce groupe de la relation pure ne peut donc ni choisir les "projets communs»—-valorisés par les communautaires, ni la passion pour la raison qui vient d'être exprimée, ni enfin le fondement de la famille, signe d'un engagement temporel. Il leur reste une seule réponse possible, «le fait d'être proche» de leur partenaire, c'est ce qu'ils font en effet un peu plus. Cela semble paradoxal puisque les individus qui, volontairement, préferrent avoir une certaine distance vis-à-vis de leur conjoint sont ceux qui déclarent être "proches». S'ils retiennent ce terme de proximité relationnelle, c'est parce que, dans la liste proposée, ils le préferent à celui du rapprochement par la création d'une «famille». À la question sur les qualités des conjoints, ce type de proximité n'est pas valorisé. En effet, les partisans de l'intimité personnelle n'estiment pas que leur partenaire est plus «complice» que les partisans des deux autres groupes; ils ne demandent pas plus «quelqu'un qui écoute et comprenne». Ils déclarent seulement attacher un peu plus d'importance à ce que leur conjoint «fasse bien l'amour avec» eux $^{16}$ : la relation sexuelle est le symbole de l'union, sans pour autant se confondre avec elle puisque la fidélité est sans doute moins exigée que dans les autres $\operatorname{groupes}^{17}$. On perçoit un embarras dans le groupe de l'intimité personnelle qui éprouve des difficultés à nommer la relation souhaitée. Le terme que Giddens retient, "pure», reflète aussi, nous semble-t-il, une telle attitude: pure de quoi? De tout partage? Il existe une contradiction inhérente à la formation de toute relation: pour que deux individus soient ensemble, ils doivent mettre en commun certains territoires personnels et donc objectivement ne plus en avoir la propriété exclusive. Tout lien repose sur un certain renoncement à soi, un soi entièrement indépendant.

\section{CONCLUSION. LA LÉGITIMITÉ DE L'INTIMITÉ PERSON NELLE ET LA SÉCURITÉ PERSON NELLE}

Les différences entre les modèles retenus ne doivent pas faire disparaître le problème commun qu'ils ont à résoudre. Tous doivent rendre légitime la «séparation» ponctuelle, le temps ou le territoire personnel qu'un des partenaires veut préserver ou prendre. La force du principe de l'indépendance et de l'autonomie est presque toujours contrecarrée par une autre force, celle de la protection que réclame l'autre. Un travail précédent (De Singly, 2001b), reposant sur des entretiens approfondis auprès de jeunes couples, a montré que les tensions qui naissent dans la vie quotidienne pro-

16. Soit respectivement $39 \%$ pour la relation pure, $28 \%$ pour la communauté et $30 \%$ pour le double respect.

17. Cela demande à être vérifié empiriquement. C'est Giddens qui le prévoit. 
viennent de la manière dont l'autre, le partenaire, interprète le fait que son conjoint revendique son intimité personnelle. C'est ainsi que le travail professionnel apporté à la maison pour être effectué le soir n'est pas nécessairement mal vécu par le partenaire contrairement à un long coup de téléphone à un ami. La légitimité du travail professionnel et celle de la relation amicale, prise sur un temps considéré comme devant être commun, different. Du point de vue du partenaire, la «séparation» pendant un temps limité peut être vécue comme normale, mais elle peut être perçue au contraire comme menaçante pour la relation et pour soi. Elle devient une "offense».

Deux formes d'offense territoriale doivent être distinguées: celle que souligne Goffman (1973), un envahissement d'un territoire personnel par quelqu'un qui n'est pas autorisé à pénétrer (le viol étant le cas le plus exemplaire); celle, trop souvent oubliée, qui consiste à éviter de partager un territoire. Dans un roman de John O’Farrell (2001), le héros marié, qui travaille à son compte, passe de nombreuses heures dans son bureau. Il fuit ses jeunes enfants qui le fatiguent et son épouse qu'il trouve trop mère et pas assez aimante. Son épouse lui reproche: «Michael, ça ne me gêne pas que tu ne sois jamais là, mais ça me gêne que tu n'aies pas envie d'être là» (p. 73, souligné par l'auteur). Cette expression souligne l'importance de l'interprétation de la séparation provisoire, de la revendication d'une intimité personnelle. Pour que le couple donne satisfaction à chacun, il est nécessaire que chaque demande d'indépendance soit approuvée par l'autre. Quelle que soit son intensité, l'intimité personnelle doit toujours être construite comme légitime, en respectant trois conditions: elle doit être perçue à la fois comme utile pour celui ou celle qui la demande, comme non menaçante pour le couple, et comme ne déstabilisant pas la sécurité ontologique du partenaire. Même sous le régime du primat de l'intimité personnelle, la vie conjugale ne peut se résumer à la revendication d'être soi; elle réclame toujours la prise en compte aussi de l'intérêt de son partenaire et de l'intérêt du couple.

La relation n'est donc pas "pure» de toute dépendance, nécessaire pour assurer une des fonctions de la vie commune, contribuer à la sécurité de chacun. L'individualisation se heurte moins à la communauté qu'à la revendication, cachée dans la fusion. La femme ou l'homme contemporain ne se définit pas que par ses rêves de liberté, d'indépendance et d'autonomie. Il subit des coups et des contrecoups qui lui font apprécier un certain "confort», assuré dans la vie commune par les habitudes (Kaufmann, 1992, 1993). L'individu intériorise en lui l'incertitude de la société moderne avancée de telle sorte qu'il peut basculer dans la dépression (Ehrenberg, 1995, 1998). Il est fragilisé. Giddens sous-estime la force de ces dangers qui poussent les partenaires à inventer une relation qui ne soit pas «pure». En effet, une telle relation contient en elle-même, comme le montre l'analyse des couples homosexuels ouverts (Lerch, 2000), des menaces, des incertitudes. La prise d'indépendance, au sein de la vie de couple, peut avoir pour conséquence des blessures identitaires pour l'autre. Le besoin de "sécurité» doit être concilié avec le besoin d'expression personnelle, d'authenticité. Chacun doit apprendre à combiner le dosage entre le degré d'indépendance et la reconnaissance d'autres besoins, notamment la stabilité et la sécurité. À l'intérieur 
même du groupe des personnes ayant accompli un cycle complet d'études secondaires, et a priori plus favorables aux valeurs de l'individualisation, on a vu comment bon nombre de femmes et d'hommes pratiquent la dialectique, non pas pour satisfaire «modernité» et «tradition»-la première étant du côté du «je» et la seconde du côté $\mathrm{du}$ «nous»—, mais pour faire en sorte que la liberté n'engendre pas trop d'insécurité et de menace pour soi. C'est pour cette raison que la légitimité acceptée par les deux partenaires de telle ou telle «liberté» individuelle — inscrite dans telle ou telle pratique, effectuée sans le conjoint — est décisive. Lorsque la séparation est légitime pour les deux, ni la confiance ni le sentiment d'insécurité ne sont affectés. Reste à chacun, d'une part, à pondérer le poids des pratiques communes et des pratiques séparées et, d'autre part, à convertir son partenaire au fait que la séparation ponctuelle n'est pas annonciatrice, bien au contraire, d'une séparation totale.

\section{RÉSUMÉ}

Il s'agit dans cet article de soumettre à la critique, à la fois théorique et empirique, le modèle de la «relation pure», proposé par Giddens comme forme idéale de la vie à deux dans les sociétés modernes avancées. Ce modèle sous-estime nettement le besoin de sécurité ontologique des individus, même les plus «individualisés ». La fragilité de ces derniers réclame certaines conditions, et notamment des preuves de reconnaissance de soi qui s'inscrivent dans la demande — qui reste très forte-de fidélité, ou encore de temps passé ensemble. Une enquête par questionnaire passé auprès de membres de couples de la région parisienne et d'un certain niveau scolaire permet de montrer la force des compromis entre la «pureté » des libertés individuelles au sein de la vie conjugale et les contraintes de la reconnaissance de chacun des deux partenaires. La vie commune n'a donc pas nécessairement comme avenir «la relation pure».

\section{RESUMEN}

Se trata en este artículo de someter a la crítica, a la vez teórica y empírica, el modelo de la «relación pura» propuesta por Giddens, como forma ideal de la vida a dos en las sociedades modernas avanzadas. Este modelo subestima claramente la necesidad de la seguridad ontológica de los individuos, aún los más «individualizados ». La fragilidad de éstos últimos exige ciertas condiciones, y principalmente de las pruebas de reconocimiento de sí que se inscriben en la demanda - que queda muy fuerte — de fidelidad, o aún del tiempo pasado juntos. Una investigación por cuestionario realizado a miembros de parejas de la región parisina y con un cierto nivel de escolaridad permite mostrar la fuerza de los compromisos entre la «pureza » de las libertades individuales en el seno de la vida conyugal y las limitaciones del reconocimiento de cada uno de los dos compañeros. La vida común no tiene por tanto, necesariamente como el porvenir, «la relación pura».

\section{SUMMARY}

This article is about subjecting to criticism, both theoretically and empirically, the model of the "pure relationship", put forward by Giddens as the ideal form of shared life in advanced modern societies. This model clearly underestimates the need for individuals, even the most "individualized", to feel ontologically safe. Their frailty calls for certain conditions and in particular 
proof of self-recognition which is part of the claim — which remains strong — of faithfulness or even of more time spent together. A questionnaire, given to members of couples from the Paris region having obtained a certain level of education, shows the strength of compromises between the "purity" of individual freedoms in married life and the constraints due to the recognition of the other's presence by each of the two partners. Thus, the future of married life is not necessarily found in that of the "pure relationship."

\section{BIBLIOGRAPHIE}

ArIÈs, P. (1960), L'enfant et la vie familiale sous l'ancien régime, Paris, Plon.

Beck, U. et E. Beck-Gernsheim (1990), Das ganz normale Chaos der Liebe, Frankfurt am Main, Suhrkamp.

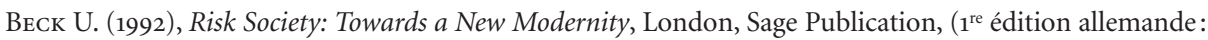
1986).

Berger, P. et H. Kellner (1988), «Le mariage et la construction de la réalité», Dialogue, nº 102, p. 6-23

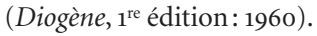

Chaumier, S. (1999), La déliaison amoureuse, Paris, A. Colin.

Corbin, A. (1987), «Coulisses», in Perrot, M. (dir.), De la Révolution à la Grande Guerre, tome IV, Histoire de la vie privée (Ariès, P., Duby, G., éds.), Paris, Seuil, p. 413-611.

Durkheim, E. (1906), «Le divorce par consentement mutuel», Revue bleue, 44, 5.

Ehrenberg, A. (1995), L'individu incertain, Paris, Calmann-Lévy.

Ehrenberg, A. (1998), La fatigue d'être soi, Paris, O. Jacob.

Eleb, M. et A. Debarre-Blanchard (1989), Architectures de la vie privée: maisons et mentalités, XVII ${ }^{e}$-XIX ${ }^{e}$ siècles, Bruxelles, AAM éditions.

Élias, N. (1973), La civilisation des moeurs, Paris, Calmann-Lévy (1 $1^{\mathrm{re}}$ éd.: 1939).

Finkenauer, C. et H. Hazam (2000), «Disclosure and secrecy in marriage: Do both contribute to marital satisfaction", Journal of Social and Personal Relationships, vol 17, 2, p. 245-263.

Gagnon, S. (1993), «Une critique des sens de l'intime», in Brunet, M., Gagnon, S. (eds.), Discours et pratiques de l'intime, Québec, IQRC, p. 245-254.

Giddens, A. (1987), La constitution de la société, Paris, Presses universitaires de France.

Giddens, A. (1991), Modernity and Self-Identity. Self and Society in the Late Modern Age, Stanford, Stanford University Press.

Giddens, A. (1992), The Transformation of Intimacy. Sexuality, Love and Eroticism in Modern Societies, Stanford, Stanford University Press.

Giddens, A. (1993), «Identité de soi, transformation de l'intimité et démocratisation de la vie», in Audet, M., Bouchikhi, H. (eds.), Structuration du social et modernité avancée. Autour des travaux d'Anthony Giddens, Sainte-Foy, Presses de l'Université Laval, p. 455-495.

Goffman, E. (1973), La mise en scène de la vie quotidienne. Les relations en public, Paris, Minuit (Relations in Public).

Hall, C. (1987), «Sweet home», in Perrot, M. (dir.), De la Révolution à la Grande Guerre, tome IV, Histoire de la vie privée (Ariès, P., Duby, G., dir.), Paris, Seuil, p. 42-87.

Jamieson, L. (1999), «Intimacy transformed? A critical look at the "pure relationship" », Sociology, 33, 3, p. 477-494.

Kalmijn, M. et W. Bernasco (2001), «Joint and Separated Lifestyles in Couple Relationships», Journal of Marriage and the Family, vol. 63, 3, p. 639-654.

Kaufmann, J.-C. (1992), La trame conjugale. Analyse du couple par son linge, Paris, Nathan.

Kaufmann, J.-C. (1993), Sociologie du couple, Paris, Presses universitaires de France.

Kaufmann, J.-C. (2001), Ego. Pour une sociologie de l'individu, Paris, Nathan.

Kellerhals, J., J.-F. Perrin, G. Cresson, L. Voneche et G. Wirth (1982), Mariages au quotidien, Lausanne, P.-M. Favre. 
Knapp, J.-J. et R.-N. Whitehurst (1981), «Le mariage et les relations sexuellement ouverts. Données et perspectives", in Murstein, I. (ed.), Styles de vie intime, Bruxelles, Pierre Mardaga, p. 34-39.

Lerch, A. (2000), L'éthique conjugale gaie à l'épreuve du multipartenariat, Dea ens-Ulm-eHess (dir. F. de Singly).

Mana (1997), numéro 3 «Approches sociologiques de l’intime» (dir. D. Le Gall).

Martin, O. et F. de Singly (2002), «Le téléphone portable dans la vie conjugale: retrouver un territoire personnel ou maintenir le lien conjugal», Réseaux, n 20 , p. 211-248.

Millet, C. (2001), La vie sexuelle de Catherine M., Paris, Seuil.

Morgan, D. (1991), «Ideologies of Marriage and Family Life», in Clark D. (ed.), Marriage, Domestic Life and Social Change: Writings for Jacqueline Burgoyne, London, Routledge.

Morgan, D. (1996), Family Connections: An introduction to Family Studies, Cambridge, Polity Press.

O'Farrell (2001), Un mec parfait (The Best a Man Can Get, Doubleday), Paris, Plon.

Passeron, J.-C. (1991), Le raisonnement sociologique, Paris, Nathan.

Renaut, A. (1989), L'ère de l'individu. Contribution à une histoire de la subjectivité, Paris, Gallimard.

Roussel, L. (1989), La famille incertaine, Paris, O. Jacob.

Simmel, G. (1999), Sociologie. Études sur les formes de socialisation, Paris, Presses universitaires de France

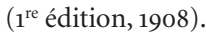

SingLY, F. de (1987), Fortune et infortune de la femme mariée, Paris, Presses universitaires de France (rééd., 2002).

Singly, F. de (1992), L'enquête par questionnaire, Paris, Nathan.

Singly, F. de (1996), Le Soi, le couple et la famille, Paris, Nathan.

SingLY, F. de (2000a), «Sur la crise de la vie conjugale», in Michaud, Y. (ed.), Qu'est-ce que la société?, tome 3, Université de tous les savoirs, Paris, O. Jacob, p. 481-491.

Singly, F. de (200ob), Libres ensemble. L'individualisme dans la vie commune, Paris, Nathan.

Singly, F. de (2001a), "Pour un "individu individualisé" ", préface, in de Singly, F. (ed.), Être soi parmi les autres. Famille et individualisation, tome 1, Paris, L'Harmattan, p. 5-14.

SingLY, F. de (2001b), «Charges et charmes de la vie privée», in Laufer, J., Marry, C., Maruani, M. (eds.), Féminin-Masculin: question pour les sciences de l'homme, Paris, Presses universitaires de France, p. 149167.

Singly, F. de et K. Chaland (2001), "Quel modèle pour la vie à deux dans les sociétés modernes avancées?», Comprendre, $\mathrm{n}^{\circ}$ 2, Le lien familial, p. 283-300.

Singly, F. de (2003), Les uns avec les autres. Quand l'individualisme crée du lien, Paris, A. Colin. 